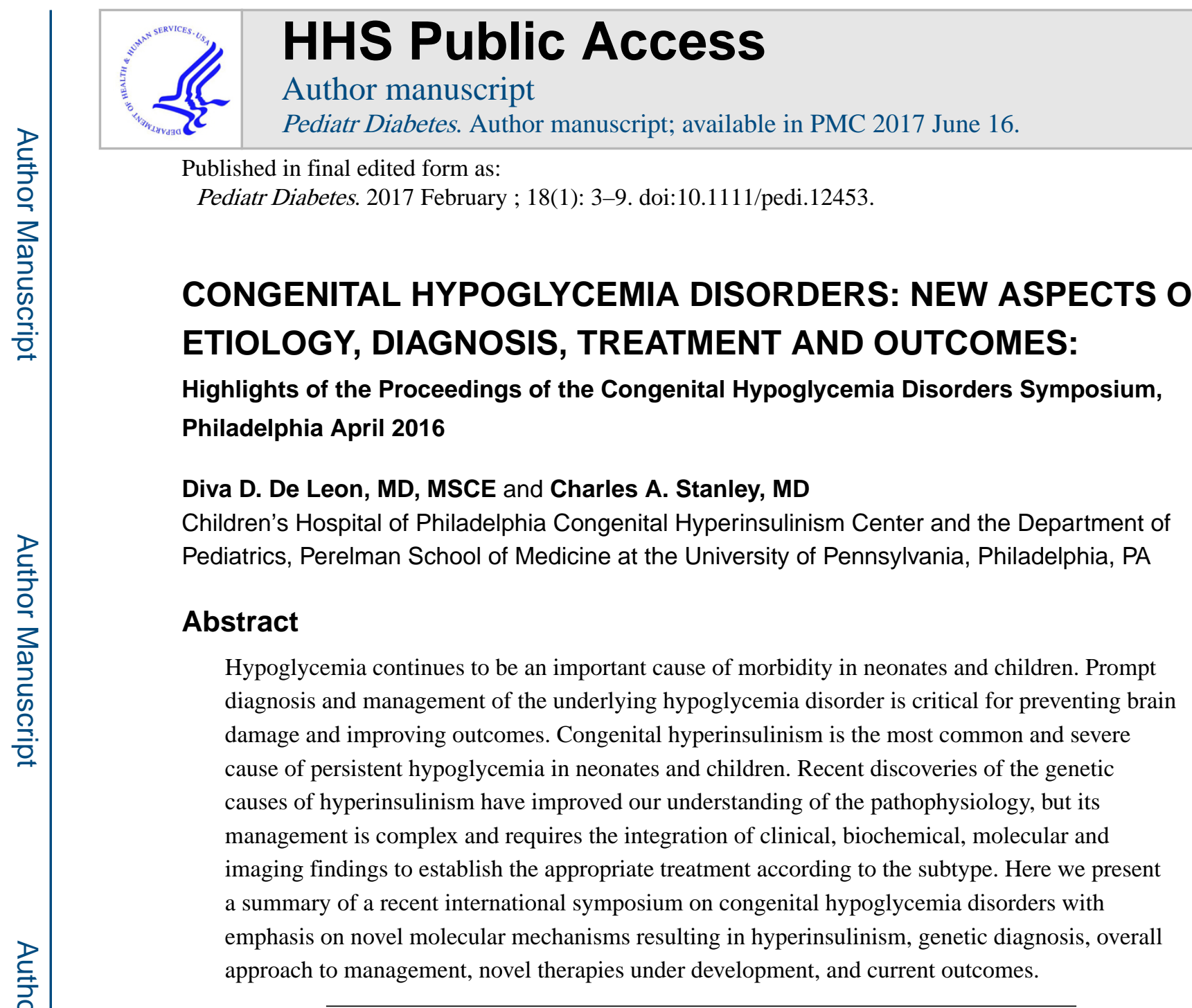

\title{
Introduction
}

Spontaneous hypoglycemia is an important cause of morbidity in neonates, infants and children. Up to $50 \%$ of children with congenital hypoglycemia disorders suffer from longterm neurodevelopmental disabilities $(1,2)$. Recent advances in the understanding of the molecular genetics and pathophysiology of these disorders have enabled improvement in outcomes through the development of early diagnostic tools and improved therapies. Moreover, the discoveries of new genetic defects involved in the pathogenesis of these disorders broadened our understanding of normal regulation of carbohydrates metabolism in humans. To review these advances and provide a platform to translate them into clinical practice, the Third International Hyperinsulinism Symposium, sponsored by The Children's Hospital of Philadelphia Congenital Hyperinsulinism Center, was held in Philadelphia Pennsylvania April 14-15, 2016.

Invited experts in molecular biology, surgery, radiology, and pediatric endocrinology represented the major pediatric hypoglycemia centers in the world. Recent advances in the etiology, diagnosis, treatment, and outcomes of congenital hyperinsulinism (HI) and of the

Corresponding Author: Diva D. De Leon, MD, MSCE, 3615 Civic Center Blvd, Abramson Research Center, Room 802A, Philadelphia, PA, 19104, Deleon@email.chop.edu, Phone: 215-590-3420, Fax: 215-590-1605. 
glycogen storage disorders (GSD) were discussed. Here we present the highlights of the aspects relating to $\mathrm{HI}$; aspects relating to GSD will be separately described in a subsequent paper.

Congenital hyperinsulinism (HI) is the most common cause of persistent hypoglycemia in infants and children. $\mathrm{HI}$ is a rare, orphan disease with an estimated incidence in the United States of 1 in 50,000 live births; incidence is significantly greater in populations with high rates of consanguineous marriage. Major advances have been made over the past two decades in understanding the molecular basis of $\mathrm{HI}(3,4)$, with many of these discoveries made by the conference speakers. Currently, mutations in eleven different genes are known to cause congenital hyperinsulinism via disturbances in the tightly controlled process by which metabolic fuels stimulate insulin secretion. Inactivating $\mathrm{K}_{\text {ATP }}$ channel mutations are the most common and severe type of HI, responsible for both focal and diffuse forms of HI. One of the most significant advances in the last decade was the development of ${ }^{18} \mathrm{~F}$-L-DOPA PET scanning to localize focal lesions and to distinguish them from diffuse forms (5). As a result, surgeons can be guided preoperatively as to the location of focal disease so that children with focal HI can now be cured by limited surgery. Concurrent with and because of better understanding of the pathophysiology and the molecular mechanisms of disease, the field is now moving towards the development of new therapeutic agents, such as GLP-1 receptor antagonists (6), mTOR inhibitors (7), and longer-acting somatostatin analogs (8).

The overall objectives of the Symposium were to: (1) educate clinicians involved in the care of infants at risk for persistent hypoglycemia on the recently published guidelines from the Pediatric Endocrine Society for evaluating and managing persistent hypoglycemia in neonates, infants and children; (2) educate clinicians involved in the care of infants and children with hypoglycemia disorders regarding recent advances in the diagnosis and management of these conditions; (3) share information about new genetic testing resources and imaging techniques being used to improve rapid diagnosis and treatment of children affected by congenital hypoglycemia disorders; (4) promote scientific dialogue between clinicians and scientists with the goal of stimulating the formulation of new research ideas and the establishment of new collaborations; and (5) to engage a new generation of clinicians and scientists and to foster their involvement in the field. This article summarizes the major topics and advances discussed at the conference.

\section{Highlights from the Conference}

\section{Congenital Hyperinsulinism - historical perspective}

Dr. Charles A. Stanley, founder of the Congenital Hyperinsulinism Center at The Children's Hospital of Philadelphia, opened the conference with a historical overview of HI. In 1954 McQuarrie (9) introduced the concepts that spontaneous occurring hypoglycemia in infants which he named "Idiopathic Hypoglycemia": 1) was likely "familial"; 2) was an important cause of brain damage; 3 ) and that there were limited treatment options for these infants. A year later, Cochrane (10) made the observation that protein feeding, specially leucine, induced hypoglycemia in some children with "idiopathic hypoglycemia", and thus, the term "leucine-sensitive hypoglycemia" was born. The development and application of the insulin assay by Berson and Yallow in 1960 (11) showed that plasma insulin concentrations were 
elevated in leucine-sensitive idiopathic hypoglycemia of infancy. The first specific treatment for $\mathrm{HI}$ evolved from the realization that diazoxide, an antihypertensive drug, could cause hyperglycemia in normal individuals. Thus, in 1964, Drash and Wolff (12) published the first account of the use of diazoxide to treat children with "leucine-sensitive hypoglycemia". The term congenital hyperinsulinism was proposed in 1976 by Stanley and Baker, who outlined the diagnostic criteria for HI: 1) hyperinsulinemia; 2) hypoketonemia; 3) hypofatty acedemia; 4) hyperglycemic response to glucagon (13). In 1989, Brunelle and colleagues (14) described diffuse and focal forms of $\mathrm{HI}$ based on selective venous pancreatic sampling, and, in 2006, the utility of ${ }^{18} \mathrm{~F}$ L-DOPA PET to localize focal HI lesions was demonstrated (15). The first genetic defects associated with HI were discovered in 1995 (16); since then, the number of known genes associated with $\mathrm{HI}$ has grown to over 11. Of all the various genetic forms of $\mathrm{HI}$, inactivating $\mathrm{K}_{\mathrm{ATP}}$ channel mutations represent the most common defects and account for $\sim 60 \%$ of all identifiable mutations in one large series (17). In addition, $\mathrm{K}_{\mathrm{ATP}}$ mutations causing $\mathrm{HI}$ account for $85 \%$ of diazoxide-unresponsive $\mathrm{HI}$ cases; these can be focal or diffuse, in approximately equal proportions. However, HI due to milder dominant $\mathrm{K}_{\mathrm{ATP}}$ mutations must also be considered in diazoxide-responsive cases as these mutations account for $17 \%$ of diazoxide-responsive cases. With this better understanding of the molecular genetics of congenital HI a "personalized medicine" approach to management is starting to emerge according to the genetic type of $\mathrm{HI}$, and particularly, according to the likelihood of a surgically-curable focal HI lesion.

\section{Management Approach}

Dr. Diva De León, director of The Children's Hospital of Philadelphia Congenital Hyperinsulinism Center, reviewed the current approach to the management of neonates and infants with congenital HI (Figure 1). A key clinical feature is whether the $\mathrm{HI}$ is responsive to diazoxide, since this drug acts by opening $\mathrm{K}_{\mathrm{ATP}}$ channels and is ineffective in most diffuse or focal forms of $\mathrm{K}_{\mathrm{ATP}} \mathrm{HI}$. In highlighting the importance of clearly defining diazoxide responsiveness, Dr. De León proposed that: "if after at least 5 days of treatment with diazoxide at a maximal dose of $15 \mathrm{mg} / \mathrm{kg} / \mathrm{day}$, an infant continues to require intravenous dextrose support and/or is unable to maintain normal plasma glucose, not only during a normal feeding schedule but also during an overnight fast, then, the infant should be considered diazoxide unresponsive". In diffuse disease, biallelic recessive mutations in the genes encoding either one of the two components of the $\mathrm{K}_{\mathrm{ATP}}$ channel ( $A B C C 8$ or $K C N J 11$ ) or monoallelic dominant mutations result in lack of expression of $\mathrm{K}_{\mathrm{ATP}}$ channels on the plasma membrane of all pancreatic beta cells.

While in focal disease, a monoallelic recessive mutation inherited from the father coupled with somatic loss of the maternal $11 \mathrm{p}$ chromosomal region encoding the $\mathrm{K}_{\mathrm{ATP}}$ channel as well as tumor suppressor genes, results in a focal area of adenomatosis with beta cells lacking functional $\mathrm{K}_{\mathrm{ATP}}$ channels. Therefore, for children with diazoxide-unresponsive HI, molecular diagnosis to identify a paternally-transmitted recessive $\mathrm{K}_{\mathrm{ATP}}$ mutation, followed by ${ }^{18} \mathrm{~F}$ L-DOPA PET scanning, should be pursued without delay to rule out a focal lesion, which could be resected. 


\section{Novel Mechanisms of $\mathrm{HI}$}

a. $\quad$ HI in Beckwith Wiedeman syndrome (BWS): Dr. Jennifer Kalish, from The Children's Hospital of Philadelphia, discussed the clinical spectrum of HI in BWS and the relationship of the underlying genetic and epigenetic mechanisms of BWS to the severity of the HI. Hypoglycemia due to HI occurs in approximately $50 \%$ of BWS cases; in the majority of cases, the $\mathrm{HI}$ is mild and transient, but in $\sim 5 \%$ of case, HI can be severe and persistent. The BWS locus on 11 p15.5 includes two adjacent regions of imprinted genes, including IGF2, a growth promoting protein, and two growth-inhibitory genes, the non-coding RNA $H 19$ and $C D K N 1 C$, which encodes the cell cycle regulator p57 (Figure 2). In half of BWS cases, the cause is isolated hypomethylation of the imprinted control region 2 (IC2), leading to loss of expression of $C D K N 1 C$ and the adjacent voltage-gated potassium channel $K C N Q 1$; in another 5-10\% of cases, the cause is isolated hypermethylation of IC1, leading to loss of expression of $H 19$ and biallelic expression of IGF2; the remaining $20 \%$ of cases are caused by chromosome $11 \mathrm{p}$ paternal uniparental isodisomy (patUPD), leading to loss of expression of $C D K N 1 C$ and $K C N Q 1$ and to over-expression of IGF2. In the latter group, HI can be particularly severe and is especially likely to be diazoxide-unresponsive. Histologic and functional evaluation of pancreatic islets from the 11p patUPD cases suggest that the mechanism of $\mathrm{HI}$ involves both expansion of $\beta$-cell mass (adenomatosis), as well as intrinsic functional abnormalities in beta-cell insulin secretion (18).

b. Kabuki Syndrome and HI: The pathophysiology and clinical phenotype of children with HI and Kabuki syndrome was presented by Dr. Jean Baptiste Arnoux, from Hopital Universitaire Necker in Paris. After BWS, Kabuki syndrome is the second most common syndromic form of HI. Kabuki syndrome (19) is caused by inactivating mutations in two genes: autosomal recessive mutations in KMT2D (Lysine-specific methyltransferase 2D), which account for $70-75 \%$ of cases, and X-linked mutations in KDM6A (Lysine-specific demethylase $6 A$ ), which account for 1-9\% of cases. These two genes encode a DNA methyltransferase and demethylase, respectively. Five cardinal features of Kabuki syndrome include: 1) the typical "Kabuki Mask" facial characteristics (long palpebral fissure, arched eyebrows, eversion of the external third of the lower lid); 2) skeletal abnormalities; 3) dermatoglyphic abnormalities; 4) neurodevelopmental delay and intellectual disabilities; 5) postnatal growth retardation. Endocrine manifestations include growth hormone deficiency (22\% of cases), hypothyroidism (up to $10 \%$ of cases), premature thelarche (20\%), and neonatal hypoglycemia (11\%). Up to $70 \%$ of children with Kabuki syndrome have congenital $\mathrm{HI}$ and most of these are diazoxide-responsive.

c. $\quad$ Congenital Hyperinsulinism Caused by Heterozygous Uncoupling Protein 2 (UCP2) Mutations presented by Dr. Christine T. Ferrara (CHOP and UCSF). Dominant inactivating mutations in UCP2 had been described in 2008 in two patients with diazoxide-responsive $\mathrm{HI}$, but no additional cases have since been reported. New data on the function of UCP2 demonstrate that it is a key regulator 
of TCA cycle intermediates: deficiency of UCP2 may trigger excessive glucose stimulated insulin secretion by enhancing glucose oxidation. Screening of 118 infants with diazoxide-responsive HI seen between 1997-2010 identified UCP2 missense mutations in 3 unrelated infants (i.e., 3\% of all diazoxide-responsive cases). All 3 were of African-American ethnicity and presented with HI prior to 6 months of age. One mutation was previously reported (p.A268G) and two were novel (p.G61S, p.S47N). The A268G and S47N mutations had low-level frequency in sub-Saharan Africans (0.01\% and 0.3\%, 1000 Genome Database). In vitro expression studies showed reduced UCP2 activity from 20-40\% to 60$80 \%$ of normal. One case was responsive to diazoxide therapy; diazoxide was only partially effective in the other 2 cases, but coexisting risk factors may have exaggerated the severity of their HI (SGA birth weight and a possible MODY3 mutation). Hypoglycemia was thought to resolve by the age of 7.5 years in case 1 and by 11 months in case 2; however, Case 3 still required diazoxide therapy at 5 years of age. Together with the 2 patients reported in 2008, there are now a total of 5 cases of UCP2 inactivating mutations as a dominant form of diazoxideresponsive $\mathrm{HI}$. In the $\mathrm{CHOP}$ series, $\mathrm{UCP} 2$ inactivating mutations occurred at a frequency similar to other rare genetic causes of HI. Whether hypoglycemia is a problem only in early infancy that may improve or resolve later in childhood remains uncertain.

d. Hypoglycemia Due to Lower Threshold of Glucose-Stimulated Insulin Secretion in Phosphoglucomutase 1 Deficiency, was presented by Dr. Amanda M. Ackermann (CHOP). Phosphoglucomutase 1 (PGM1) deficiency is a rare disorder with a wide phenotypic spectrum, including cleft palate, hepatopathy, myopathy, short stature, and hypoglycemia in up to $75 \%$ of patients. PGM1 catalyzes the interconversion of glucose-6-phosphate and glucose-1-phosphate and is important for glycogen synthesis, glycogenolysis, and protein glycosylation. PGM1 deficiency has been categorized as both a glycogen storage disease and as a congenital disorder of glycosylation. The authors identified both fasting ketotic hypoglycemia and post-glucose load hypoketotic hyperinsulinemic hypoglycemia as features of PGM1 deficiency in one affected child. While fasting ketotic hypoglycemia is readily explained by PGM1's known roles in glycogen synthesis and glycogenolysis, the etiology of the hyperinsulinemic hypoglycemia was unknown. Since hyperinsulinemic hypoglycemia was observed after both oral and intravenous glucose challenge, gut incretins were not the cause. Supplementation with galactose, which improved but did not normalize the patient's protein glycosylation pattern, did not improve the hyperinsulinemic hypoglycemia. Therefore, the authors hypothesized that PGM1 activity within pancreatic islets modulates glucosestimulated insulin secretion (GSIS). Using mouse and human pancreatic islets, the authors demonstrated that PGM1 inhibition increased intracellular calcium levels in $\beta$ cells and lowered the glucose threshold for GSIS. The authors propose that PGM1 normally functions in $\beta$ cells to divert glucose-6-phosphate to glucose-1-phosphate, rather than entering glycolysis, in order to tightly regulate the GSIS threshold. Thus, PGM1 appears to be a novel regulator of $\beta$ cell GSIS. 
These results suggest that PGM1 might be a drug amenable target to increase $\beta$ cell insulin production in patients with diabetes.

e. Unrecognized Incidence of Congenital HI in Turner Syndrome, was presented by Dr. Christopher E. Gibson (CHOP). Congenital HI in Turner syndrome (TS) infants has been reported 6 times in the past 36 years, suggesting an increased incidence of HI in TS. KDM6A (Xp11.2) is a possible connection between HI and the $\mathrm{X}$ chromosome since mutations of this gene are associated with Kabuki syndrome and HI (see above lecture by Dr. Arnoux). The authors examined the HI patients seen at CHOP from 1974 to 2015 for TS and for possible KDM6A mutations or haploinsufficiency. They identified 8 infant girls with HI who also had TS and were treated with diazoxide, frequent feeds or sub-total pancreatectomy. Karyotyping showed $46, \mathrm{X}$ in 3 cases and mosaicism for a marker or ring $\mathrm{X}$ in the other 5. Four of the marker $\mathrm{X}$ cases lost KDM6A. The fifth girl retained $K D M 6 A$ and had no detectable KDM6A mutations; peripheral blood showed $15-20 \%$ of her cells to be 46 ,X. Functional studies of islets isolated at the time of pancreatectomy in one case showed abnormally elevated cytosolic calcium and stimulation of insulin release by a mixture of amino acids as well as by glyburide; these results suggested that $\mathrm{K}_{\mathrm{ATP}}$ channels were intact, but functionally defective. Control human islets treated with a $K D M 6 A$ inhibitor showed similar abnormalities of insulin release. These results indicate a nearly 25-fold increased incidence of HI in TS infants: 6 TS cases out of 678 total HI cases (1:110) compared to the expected incidence of TS of 1 in 2,500 newborns. The authors speculate that the HI in TS may be caused by KDM6A haploinsufficiency.

f. Syndromic Hypoketotic, Hypoinsulinemic Hypoglycemia due to a Mosaic Activating Phosphatidylinositol 3-kinase (PI3K) Mutation, presented by Dr. Sebastian Kummer, University Children's Hospital, Duesseldorf. In contrast to hypoglycemia due to congenital hyperinsulinism, there are patients with a similar metabolic profile of hypoketotic hypoglycemia, but low insulin levels and low glucose requirements to maintain euglycemia. For example, five patients with activating mutations in the insulin signal-transducing kinase AKT2 have been described, each showing a syndromic phenotype including hemihypertrophy. The authors described a 3.5 year-old girl with similar metabolic and syndromic features, but no AKT2 mutation, suggesting a possible mutation in another gene of the insulin signaling pathway. Exome sequencing revealed a mosaic mutation (p.Glu726Lys) in PIK3CA, encoding the catalytic subunit of phosphatidylinositol 3-kinase that was detected in lymphocyte, hair bulb, fibroblast, and cheek swab DNA from the patient but neither parent. Mosaic mutations in this gene can cause segmental overgrowth disorders such as the Megalencephaly-Capillary malformation (MCAP) syndrome, but have not been described to be associated with hypoglycemia so far. Features of MCAP syndrome were found in the patient. Although glucose requirements to maintain euglycemia in the patient were relatively low $(2.4 \mathrm{mg} / \mathrm{kg} / \mathrm{min})$, fasting tolerance was very short $(\sim 2.5-3 \mathrm{~h}$, aged 3 years). Increased basal levels of signaling downstream from PI3K were 
detected in serum starved dermal fibroblasts from the patient. The authors suggest that their patient's hypoglycemic can be explained by liver involvement with somatic mosaicism of the PIK3CA mutation leading to suppressed hepatic gluconeogenesis, driven by constitutive, ligand-independent activation of the insulin receptor pathway.

\section{New Insights into Molecular Diagnosis}

a. Dr. Arupa Ganguly, from the University of Pennsylvania, reviewed her laboratory's approach to molecular diagnosis of HI using Sanger sequencing as the first tier test to identify mutations in $A B C C 8$ or $K C N J 11$, and next generation sequencing for the remaining $8 \mathrm{HI}$ genes using the Ion-Torrent PGM platform for second tier testing. The finding of a single heterozygous recessive mutation in either $A B C C 8$ or $K C N J 11$ had a positive predictive value of $94 \%$ for focal disease; this was increased to $98 \%$ if the mutation was inherited from the father. In contrast, two recessive $\mathrm{K}_{\mathrm{ATP}}$ channel mutations were predictive of diffuse disease (17). The development of next generation sequencing for molecular diagnosis in HI promises to increase detection of mutations that are in noncoding regions and promoter regions or that are expressed at low levels of mosaicism. Dr. Ganguly also discussed limitations in the accuracy of "in-silico" prediction software, e.g., SIFT and PolyPhen, to identify pathogenic variants: these programs correctly identified pathogenic variants with a sensitivity of $96 \%$, but with only a poor specificity of $69 \%$.

b. Dr. Sian Ellard, from the University of Exeter, shared her laboratory's extensive experience with genetic testing for HI. Characteristics essential for genetic diagnosis in $\mathrm{HI}$ are that it should be fast, accurate, and comprehensive for every patient, because it guides treatment and defines the risk of hyperinsulinism for siblings and future offspring. Dr. Ellard highlighted the importance for global sharing of genetic variant data across disorders and countries to improve the interpretation of novel variants. She reviewed a stepwise approach to genetic testing of HI, starting with Sanger sequencing for mutations in $A B C C 8$ and $K C N J 11$, followed by targeted next generation sequencing of all known hyperinsulinism genes, and, for the negative cases, the use of whole exome or genome sequencing to drive the discovery of new genetic causes of HI. Through the use of whole exome sequencing, Dr. Ellard has discovered a de novo variant in $C A C N A 1 D$, encoding the alpha subunit of the L-type calcium channels, in a child with $\mathrm{HI}$ and neurodevelopmental deficits. The missense mutation was previously reported in individuals with a phenotype characterized by primary aldosteronism, seizures and neurological abnormalities. Functional studies demonstrated that this is a gain-of-function mutation. This discovery may have important therapeutic implications for affected individuals since they may benefit from treatment with calcium channel blockers. 


\section{Novel Investigational Therapies}

Exciting advances in the development of new medical therapies presented at The Symposium.

a) Exendin-(9-39)—Dr. Diva De León, from The Children's Hospital of Philadelphia Congenital Hypeirnsulinism Center, discussed the development of exendin-(9-39) (6, 20), a GLP-1 receptor antagonist, for the treatment of congenital HI. Data from preclinical studies demonstrated that the GLP-1 receptor is constitutively activated in pancreatic islets lacking $\mathrm{K}_{\text {ATP }}$ channels, and that exendin-(9-39) inhibits insulin secretion in mouse and human islets with $\mathrm{K}_{\mathrm{ATP}}$ channels defects and increases fasting glucose in a mouse model of $\mathrm{K}_{\mathrm{ATP}} \mathrm{HI}$. In a proof-of-concept study, Dr. De León and colleagues demonstrated that continuous intravenous infusion of exendin-(9-39) increased fasting glucose in adolescents and adults with $\mathrm{K}_{\mathrm{ATP}} \mathrm{HI}$. More recently, they showed that exendin-(9-39) prevents protein-induced hypoglycemia in children with $\mathrm{K}_{\mathrm{ATP}} \mathrm{HI}$. An ongoing clinical trial is evaluating the effects of a single intravenous dose of exendin-(9-39) on glucose requirements of infants with diazoxide-unresponsive $\mathrm{HI}$.

b) Sirolimus-Dr. Khalid Hussain, from Great Ormond Street Hospital for Children, London, presented their experience with sirolimus (7), an mTOR inhibitor currently approved for the prophylaxis of organ rejection in patients age 13 years and older receiving kidney transplantation. Based on evidence from histophatologic studies showing increased $\beta$-cell proliferation, upregulation of growth promoting factors, and that the mTOR pathway is constitutively activated in pancreas of children with congenital hyperinsulinism (21), Dr. Hussain's group had previously published an account of 4 infants with medicallyunresponsive hyperinsulinism that were treated with sirolimus preventing the need for pancreatectomy. Dr. Hussain indicated that they have now treated a total of 15 patients with sirolimus, either alone or in combination with octreotide. In 4 patients, sirolimus was discontinued because they had been switched to lanreotide. In 5 patients there was no improvement in glycemic control while on sirolimus. Although there were no clear genotype/phenotype correlations in terms of the response to treatment with sirolimus, Dr. Hussain stated that in patients with $\mathrm{K}_{\mathrm{ATP}}$ mutations, it appeared that patients with homozygous mutations had the least response, while patients that were compound heterozygous had a favorable response, and patients with dominant mutations had slightly better response. Dr. Hussain emphasized the need for a controlled clinical trial to better evaluate the response to sirolimus.

c) Continuous Glucagon-Dr. Susan Empting from Otto-von-Guericke University in Germany, discussed the use of continuous glucagon infusion for the treatment of congenital HI. The use of intravenous administration of glucagon to reduce the requirement for glucose support is common practice, however, outpatient management of HI with glucagon is limited by the lack of a soluble formulation of glucagon that would allow long-term continuous subcutaneous administration. To overcome this limitation, Xeris Pharmaceuticals has developed a soluble, stable glucagon formulation for continuous subcutaneous administration that is now being evaluated in a proof-of-concept clinical trial in infants with diazoxide-unresponsive HI. 
d) Insulin Receptor Inhibitory Antibody-The last presentation from this session was from Dr. Diva De León, who presented preclinical studies examining the effect of an insulin receptor antibody on glucose metabolism in the mouse model of $\mathrm{K}_{\text {ATP }}$ HI. This fully human allosteric IgG2 monoclonal antibody acts as an allosteric inhibitor of insulin-dependent activation of the insulin receptor (XOMA Corporation). Dr. De León shared data from an experiment in which the antibody was administered twice weekly to $S U R 1^{-/-}$and wild type mice. Treatment with the insulin receptor antibody significantly increased fasting glucose. Xoma has completed a phase 1 study in normal individuals and a phase 2 trial is currently ongoing in individuals with congenital $\mathrm{HI}$.

\section{Prevention of Brain Damage: hypoglycemia guidelines and current neurodevelopmental outcomes}

a) Pediatric Endocrine Society Hypoglycemia Guidelines-Dr. Paul Thornton, from Cook Children's Medical Center, Fort Worth, TX, discussed the recently published Pediatric Endocrine Society Hypoglycemia Guidelines (22). These guidelines outline which neonates, infants and children should be evaluated for hypoglycemia; the investigation of persistent hypoglycemia in neonates, infants and children; and the management of neonates, infants and children with documented persistent hypoglycemia disorder. Dr. Thornton emphasized the importance of recognizing neonates at high risk for hypoglycemia, which should include, in addition to the ones included in standard guidelines, neonates who had perinatal stress, those with family history of a genetic form of hypoglycemia, and those with congenital syndromes known to be associated with hypoglycemia such as Beckwith Wiedemann syndrome and hypopituitarism. The most important message of this presentation was the recommendation to investigate and rule out a persistent hypoglycemia disorder in neonates that: (1.) have an episode of symptomatic hypoglycemia, or require intravenous dextrose to treat hypoglycemia; (2.) those neonates who continue to have preprandial plasma glucose concentrations $<50 \mathrm{mg} / \mathrm{dL}$ beyond the transitional first $48 \mathrm{hrs}$ of life; and (3.) those with a potential genetic risk. This evaluation should take place after the first $48 \mathrm{hrs}$ of life when the period of transitional adaptation for glucose regulation after birth should have resolved, and a critical sample obtained at the time of hypoglycemia could be taken to interpret and determine the underlying cause of the hypoglycemia.

b) Neurodevelopmental Outcomes-Hypoglycemia remains an important cause of brain injury in children with HI, leading to long-term neurological impairments. These poor neurodevelopmental outcomes have not changed much since the 1970's despite tremendous advances in the field. Dr. Diva De León summarized data from published studies showing that neurodevelopmental deficits are common in both transient and permanent HI (23), independent of approach to treatment, whether medically treated or surgically treated $(1,24$ 27). Dr. De León recommended that children with HI undergo formal neurodevelopmental evaluation at 12-18 months of age, at 5 years of age, and between first and third grade. These evaluations are critical to identify children that need intervention and therapy.

\section{Hyperketotic Hypoglycemia}

Dr. Joseph Wolfsdorf, Boston Children's Hospital, opened the session on hyperketotic hypoglycemia with an overview of the ketotic hypoglycemia disorders. Dr. Wolfsdorf 
emphasized the importance of differentiating between accelerated starvation, also known as "idiopathic hypoglycemia" in an otherwise normal child, and hypoglycemia with ketosis, which has a large differential diagnosis, including glycogen storage diseases, growth hormone and cortisol deficiency, and other hypoglycemic disorders. Evaluations solely focused on a critical sample at the type of hypoglycemia may miss the diagnosis of mild forms of glycogen storage disease (GSD) (type 0, VI, and IX) (28), thus, children that have at least 2 episodes of "ketotic hypoglycemia" should be carefully evaluated for the possibility of an underlying hypoglycemia disorder. Among the disorders that present with hypoglycemia and ketosis, Dr. Wolfsdorf reviewed a recently described disorder that results from inactivating mutations in the gene encoding monocarboxylate transporter 1 (MCT1). Both, homozygous and heterozygous mutation carrying children present with recurrent episodes of severe ketoacidosis and mild hypoglycemia provoked by fasting or infections (29). Dr. David Weinstein, University of Florida, closed the symposium with a comprehensive review of the natural history, diagnostic approach and treatment of GSD. This topic will be published separately in this Journal.

\section{References}

1. Lord K, Radcliffe J, Gallagher PR, Adzick NS, Stanley CA, De Leon DD. High Risk of Diabetes and Neurobehavioral Deficits in Individuals With Surgically Treated Hyperinsulinism. J Clin Endocrinol Metab. 2015; 100:4133-9. [PubMed: 26327482]

2. Melis D, Parenti G, Della Casa R, Sibilio M, Romano A, Di Salle F, et al. Brain damage in glycogen storage disease type I. J Pediatr. 2004; 144:637-42. [PubMed: 15127000]

3. Lord K, De Leon DD. Monogenic hyperinsulinemic hypoglycemia: current insights into the pathogenesis and management. Int J Pediatr Endocrinol. 2013; 2013:3. [PubMed: 23384201]

4. Stanley CA. Perspective on the Genetics and Diagnosis of Congenital Hyperinsulinism Disorders. J Clin Endocrinol Metab. 2016; 101:815-26. [PubMed: 26908106]

5. Laje P, States LJ, Zhuang H, Becker SA, Palladino AA, Stanley CA, et al. Accuracy of PET/CT Scan in the diagnosis of the focal form of congenital hyperinsulinism. J Pediatr Surg. 2013; 48:38893. [PubMed: 23414871]

6. Calabria AC, Li C, Gallagher PR, Stanley CA, De Leon DD. GLP-1 receptor antagonist exendin-(939) elevates fasting blood glucose levels in congenital hyperinsulinism owing to inactivating mutations in the ATP-sensitive K+ channel. Diabetes. 2012; 61:2585-91. [PubMed: 22855730]

7. Senniappan S, Alexandrescu S, Tatevian N, Shah P, Arya V, Flanagan S, et al. Sirolimus therapy in infants with severe hyperinsulinemic hypoglycemia. N Engl J Med. 2014; 370:1131-7. [PubMed: 24645945]

8. Le Quan Sang KH, Arnoux JB, Mamoune A, Saint-Martin C, Bellanne-Chantelot C, Valayannopoulos V, et al. Successful treatment of congenital hyperinsulinism with long-acting release octreotide. Eur J Endocrinol. 2012; 166:333-9. [PubMed: 22048969]

9. Mc QI. Idiopathic spontaneously occurring hypoglycemia in infants; clinical significance of problem and treatment. AMA Am J Dis Child. 1954; 87:399-428. [PubMed: 13147532]

10. Cochrane WA, Payne WW, Simpkiss MJ, Woolf LI. Familial hypoglycemia precipitated by amino acids. J Clin Invest. 1956; 35:411-22. [PubMed: 13306783]

11. Yalow RS, Berson SA. Immunoassay of endogenous plasma insulin in man. J Clin Invest. 1960; 39:1157-75. [PubMed: 13846364]

12. Drash A, Wolff F. Drug Therapy in Leucine-Sensitive Hypoglycemia. Metabolism. 1964; 13:48792. [PubMed: 14193979]

13. Stanley CA, Baker L. Hyperinsulinism in infants and children: diagnosis and therapy. Adv Pediatr. 1976; 23:315-55. [PubMed: 795282] 
14. Brunelle F, Negre V, Barth MO, Fekete CN, Czernichow P, Saudubray JM, et al. Pancreatic venous samplings in infants and children with primary hyperinsulinism. Pediatr Radiol. 1989; 19:100-3. [PubMed: 2537942]

15. Otonkoski T, Nanto-Salonen K, Seppanen M, Veijola R, Huopio H, Hussain K, et al. Noninvasive diagnosis of focal hyperinsulinism of infancy with [18F]-DOPA positron emission tomography. Diabetes. 2006; 55:13-8. [PubMed: 16380471]

16. Thomas PM, Cote GJ, Wohllk N, Haddad B, Mathew PM, Rabl W, et al. Mutations in the sulfonylurea receptor gene in familial persistent hyperinsulinemic hypoglycemia of infancy. Science. 1995; 268:426-9. [PubMed: 7716548]

17. Snider KE, Becker S, Boyajian L, Shyng SL, MacMullen C, Hughes N, et al. Genotype and phenotype correlations in 417 children with congenital hyperinsulinism. J Clin Endocrinol Metab. 2013; 98:E355-63. [PubMed: 23275527]

18. Kalish JM, Boodhansingh KE, Bhatti TR, Ganguly A, Conlin LK, Becker SA, et al. Congenital hyperinsulinism in children with paternal 11p uniparental isodisomy and Beckwith-Wiedemann syndrome. J Med Genet. 2016; 53:53-61. [PubMed: 26545876]

19. Bogershausen N, Gatinois V, Riehmer V, Kayserili H, Becker J, Thoenes M, et al. Mutation Update for Kabuki Syndrome Genes KMT2D and KDM6A and Further Delineation of X-Linked Kabuki Syndrome Subtype 2. Hum Mutat. 2016; 37:847-64. [PubMed: 27302555]

20. De Leon DD, Li C, Delson MI, Matschinsky FM, Stanley CA, Stoffers DA. Exendin-(9-39) corrects fasting hypoglycemia in SUR-1-/- mice by lowering cAMP in pancreatic beta-cells and inhibiting insulin secretion. J Biol Chem. 2008; 283:25786-93. [PubMed: 18635551]

21. Alexandrescu S, Tatevian N, Olutoye O, Brown RE. Persistent hyperinsulinemic hypoglycemia of infancy: constitutive activation of the mTOR pathway with associated exocrine-islet transdifferentiation and therapeutic implications. International journal of clinical and experimental pathology. 2010; 3:691-705. [PubMed: 20830240]

22. Thornton PS, Stanley CA, De Leon DD, Harris D, Haymond MW, Hussain K, et al. Recommendations from the Pediatric Endocrine Society for Evaluation and Management of Persistent Hypoglycemia in Neonates, Infants, and Children. J Pediatr. 2015; 167:238-45. [PubMed: 25957977]

23. Avatapalle HB, Banerjee I, Shah S, Pryce M, Nicholson J, Rigby L, et al. Abnormal Neurodevelopmental Outcomes are Common in Children with Transient Congenital Hyperinsulinism. Front Endocrinol (Lausanne). 2013; 4:60. [PubMed: 23730298]

24. Menni F, de Lonlay P, Sevin C, Touati G, Peigne C, Barbier V, et al. Neurologic outcomes of 90 neonates and infants with persistent hyperinsulinemic hypoglycemia. Pediatrics. 2001; 107:476-9. [PubMed: 11230585]

25. Mazor-Aronovitch K, Gillis D, Lobel D, Hirsch HJ, Pinhas-Hamiel O, Modan-Moses D, et al. Long-term neurodevelopmental outcome in conservatively treated congenital hyperinsulinism. Eur J Endocrinol. 2007; 157:491-7. [PubMed: 17893264]

26. Meissner T, Wendel U, Burgard P, Schaetzle S, Mayatepek E. Long-term follow-up of 114 patients with congenital hyperinsulinism. Eur J Endocrinol. 2003; 149:43-51. [PubMed: 12824865]

27. Steinkrauss L, Lipman TH, Hendell CD, Gerdes M, Thornton PS, Stanley CA. Effects of hypoglycemia on developmental outcome in children with congenital hyperinsulinism. J Pediatr Nurs. 2005; 20:109-18. [PubMed: 15815570]

28. Brown LM, Corrado MM, van der Ende RM, Derks TG, Chen MA, Siegel S, et al. Evaluation of glycogen storage disease as a cause of ketotic hypoglycemia in children. J Inherit Metab Dis. 2015; 38:489-93. [PubMed: 25070466]

29. van Hasselt PM, Ferdinandusse S, Monroe GR, Ruiter JP, Turkenburg M, Geerlings MJ, et al. Monocarboxylate transporter 1 deficiency and ketone utilization. N Engl J Med. 2014; 371:1900 7. [PubMed: 25390740] 


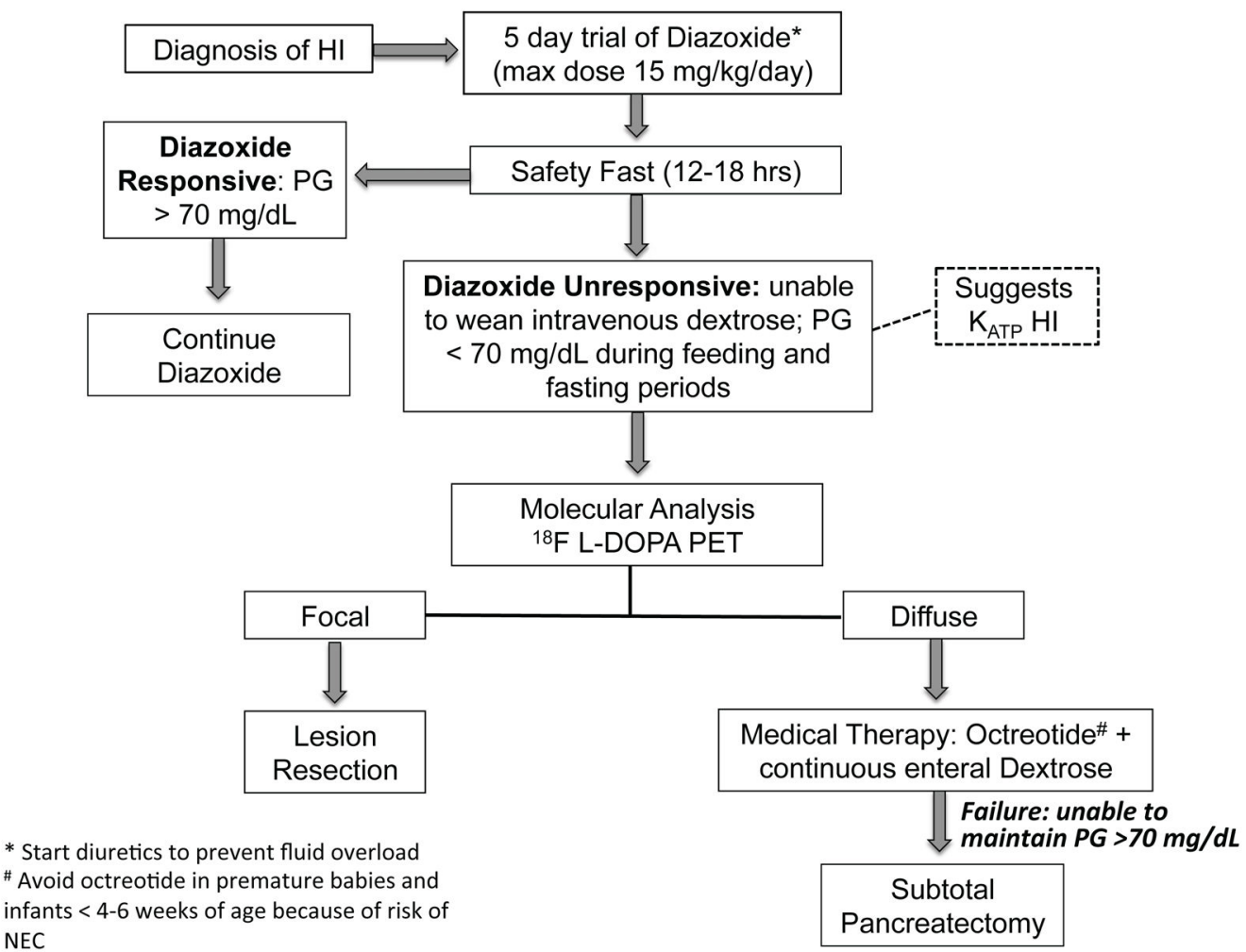

Figure 1.

Management approach to neonates, infants and children with hyperinsulinism. HI:

hyperinsulinism, PG: plasma glucose.

* Start diuretics to prevent fluid overload.

\#Avoid octreotide in premature babies and infants < 4-6 weeks of age because of the risk of necrotizing enterocolitis. 
IC2

IC1

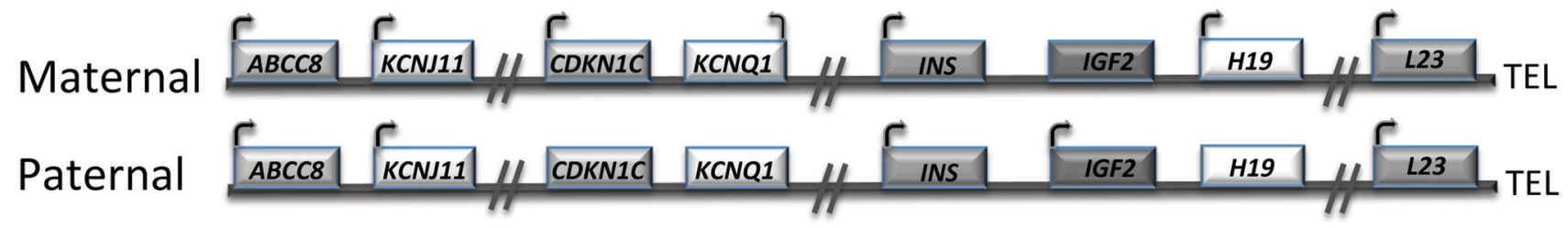

Figure 2.

Schematic representation of the normal parent of origin-specific imprinted allelic expression of the BWS locus on 11p15.5. Expressed genes are noted with an arrow. The hyperinsulinism genes $A B C C 8$ and $K C N J 11$ as well as INS and $L 23$ are biallelically expressed, while $C D K N 1 C, K C N Q 1, I G F 2$ and $H 19$ are imprinted. IC1 is methylated on the paternal allele resulting in paternal expression of $I G F 2$ and unmethylated on the maternal allele resulting in maternal expression of H19. IC2 is methylated on the maternal allele resulting in $K C N Q 1$ and $C D K N 1 C$ expression and unmethylated on the paternal allele. IC: imprinting center; TEL: telemore; $A B C C 8$ : ATP-Binding Cassette, subfamily C, member 8; $K C N J 11$ : Potassium Channel, inwardly rectifying, subfamily $\mathrm{J}$, member 11; CDKN1C: Cyclin-Dependent Kinase inhibitor $1 \mathrm{C}$; KCNQ1: Potassium Channel, voltage-gated, KQTlike subfamily, member 1; INS: insulin; IGF2: Insulin-like Growth Factor 2; L23: ribosomal protein L23-like. 